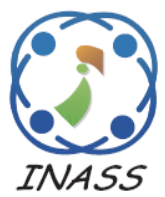

\title{
Automated Class Diagram Assessment using Semantic and Structural Similarities
}

\author{
Reza Fauzan ${ }^{1,2}$ \\ Daniel Siahaan $^{1 *}$ \\ Siti Rochimah ${ }^{1}$ \\ Evi Triandini ${ }^{3}$ \\ ${ }^{I}$ Department of Informatics, Institut Teknologi Sepuluh Nopember, Surabaya, Indonesia \\ ${ }^{2}$ Department of Electrical Engineering, Politeknik Negeri Banjarmasin, Banjarmasin, Indonesia \\ ${ }^{3}$ Department of Information System, ITB STIKOM Bali, Denpasar, Indonesia \\ * Corresponding author's Email: daniel@if.its.ac.id
}

\begin{abstract}
Class diagrams show classes in software and the relationships between those classes. A class diagram is a unified modeling language diagram commonly used in education. Thus, an assessment of class diagrams is essential for teachers who usually have students produce class diagrams based on predetermined projects. Teachers assess student-produced class diagrams based on an answer key. However, teachers have a problem with a lack of consistency in assessment as teachers can use different standards between answers. This research attempts to approach class diagram assessment automatically. The proposed approach consists of two assessments: semantic and structural similarities. Semantic similarity is calculated using lexical information in the class diagram, and structural similarity is calculated using the diagram's structure, ignoring its lexical information. Our results show that experts see semantic and structural similarities equally during assessment. The proposed approach shows substantial agreement with experts in class diagram similarity assessment. Therefore, the proposed approach can automatically assess class diagram similarity as reliably as experts can.
\end{abstract}

Keywords: Automated assessment, Class diagram, Semantic similarity, Structural similarity, UML diagram.

\section{Introduction}

Class diagrams are a part of a unified modeling language (UML) that displays a software's static structure by showing the classes within a piece of software and the logical relationships between those classes. Measurement of similarities between UML diagrams has been widely studied [1,2]; however, there are several issues with measuring class diagram similarity, such as reuse [3,4], clone detection [5-7], and assessment [8]. Class diagram reuse is useful because software engineers do not need to make diagrams from scratch, clone detection is useful when checking for plagiarism within diagrams, and assessment is useful for assisting teachers in assessing student diagram design assignments.

Several previous studies have measured similarities between two class diagrams. Park and Bae [9] began by comparing the lexical information within class diagrams, dividing lexical information into class names, relationship names, and related class information. However, lexical similarity is only calculated syntactically. The studies $[10,11]$ continued by dividing similarities into betweenstructure similarities in class names and structures. Class name similarity compares the names within each class using WordNet to find differences in semantic meaning. The structural similarity between classes compares each type of relationship, such as associations, generalizations, and dependencies. Robles [12] built an ontology domain to look for similar class diagrams. He searched for each word in the class diagram in that ontology domain. However, the comparison of words is only syntactic, and reuse is less expected in more complex class diagrams. Qiu [13] divided class diagram similarities into two categories: structural similarities and property similarities. Structural similarities are seen in lexical relationships between classes. Property similarities are seen in the lexical attributes and operations of 
each class. This study showed that combining class structures and class properties is a good approach for calculating similarity. Al-Khiaty and Ahmed [14-17] measured similarities of class properties and class diagram structures. He combined class names and information within the class (e.g., attribute and operation names) as class properties. The lexical evaluation of relations in the class diagram is used to calculate class diagram structure. Adamu $[18,19]$ built on previous studies by combining several UML diagrams. He proposed a framework for measuring similarity using the diagram's meta model [4]. He then combined the similarity of several UML diagrams using different similarity parameters [18]. Lexical information is used as a measurement parameter in class diagrams [18,20]. Yuan [21] measured structural similarity by ignoring lexical information to convert a class diagram into a graph. $\mathrm{He}$ noted that graphs could effectively measure structural similarities between class diagrams. Huang [22] also converted a class diagram into a graph. Therefore, a graph can be used to represent a class diagram.

UML diagrams can be modeled in certain forms. Based on previous studies, we can conclude that a UML diagram has two primary types of information: lexical information and structural information. Lexical information consists of all the words in the diagram, such as class names, property names, attribute names, and relation types. In contrast, structural information is the UML diagram's structure or form without any lexical information.

In this study, we focused on assessing the similarity of UML class diagrams, which are commonly used as teaching materials. Teachers give assignments to students to build class diagrams based on a project determined by the teacher. Similarity assessment is used to assist teachers in assessing the class diagrams that students build. However, teachers can have difficulty assessing class diagrams from students because class diagrams consist of many classes that have many relationships between them [23], [24]. Many components in a class diagram can affect the consistency of teacher assessments. In addition, teacher fatigue can also affect assessment consistency. Automatic assessment is a solution to maintain teacher consistency [25]. Jebli [26] attempted to assess class diagrams by obtaining lexical information from class diagrams using machine learning and ignoring diagram structure. However, structure is an important parameter for assessing UML diagram similarity [27].

In this study, we developed an approach for automatically assessing the class diagram similarities as reliably as an expert can (i.e., a teacher who conducts assessments). This study is a continuation of previous studies [28] that only assessed semantic similarity. The proposed approach divides similarity into semantic and structural similarities. We combined these two similarities to assess like an expert. We divided similarity into two because the information obtained from class diagrams consists of lexical and structural information. Our approach can show the inclination of a teacher's perspective in assessing whether to look at lexical information or class diagram structure. The tendency of perspective in assessment is obtained from the similarity weight, which has the best agreement value with the expert. The proposed semantic similarity method compares each component's lexical information using simple natural language processing (NLP) and compares within-class and between-class structures using graph edit distance (GED). The assessment of the proposed method agreement with the expert is a substantial agreement. Therefore, our approach assesses students' answers based on the answer key as reliable as an expert.

The rest of this article consists of several sections: Section 2 presents the semantic similarity assessment. Section 3 presents the structural similarity assessment. Section 4 presents the combination of previous assessment into class diagram similarity assessment. Section 5 presents the result and evaluation. Section 6 presents our discussion about the evaluation and findings. Section 7 presents the conclusion of our study.

\section{Semantic similarity assessment}

The concept of semantic similarity assessment involves seeing the similarity of two class diagrams based on lexical information obtained from those class diagrams. Information in class diagrams can be divided into property information and relationship information. This study discusses the lexical aspects of each item of information. For example, for class diagrams $\mathrm{d} 1$ and $\mathrm{d} 2$, all property information from $\mathrm{d} 1$ will be searched to find similarities to property information from $\mathrm{d} 2$. Operating information has many items, and each item from d1 will be compared with each item from d2. After obtaining the semantic similarity from each item, a greedy algorithm is needed to locate the ideal value of the previously calculated set of similarity values.

\subsection{Semantic similarity}

We determined semantic similarity using NLP [29]. We used a common NLP approach because words will be compared in one phrase consisting of two words on average. As previously explained, 


$\sum$ Tokenization $\gg$ POS Tagging $\left.\ \begin{array}{c}\text { Stopwords } \\ \text { Removal }\end{array}\right\rangle$ Lemmatization $\left.\gg \begin{array}{c}\text { Cosine } \\ \text { Similarity }\end{array}\right\rangle$

Figure. 1 Natural language processing in UML diagram semantic similarity assessment

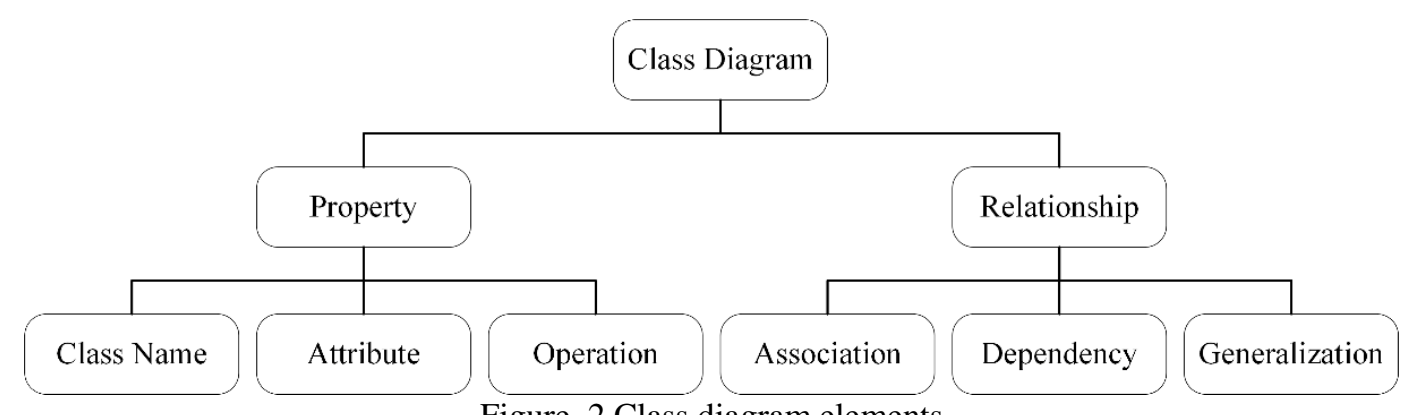

Figure. 2 Class diagram elements

semantic calculations are performed in the smallest part of each class diagram component. For example, the class name is one of the lowest parts of a class diagram, and class names have different characters from ordinary lexical information. Classes can have more than one word for a name, but the next word must be in uppercase. Therefore, NLP for semantic similarity has specific process details.

Fig. 1 shows the calculation procedure. The first part of this procedure is the tokenization process. Next, each word is given a part of speech (POS) sign. The following procedure is to eliminate stop words and lemmatization. The final part of the procedure is to calculate the similarity of words using cosine similarity.

The tokenization process involves breaking a group of words into individual units and is performed to adjust to the input words to be calculated. From the previous explanation, the lexical form of the class name, for example, getData, cannot be directly processed. Therefore, a unique method is needed for this tokenization process. First, if the next letter consists of an uppercase letter, the word will be broken up. Second, if uppercase letters are found in a sequence, the word will not be broken up until uppercase letters accompanied by lowercase letters are found.

POS tagging is intended to make the token's type known. Token types include nouns, verbs, adjectives, and so on. This process is assisted by Stanford NLP [30] using Stanford POS tagger with an English language model. Tagging uses POS from the Penn Treebank model to obtain each word's label. Before conducting the POS tagging process, all tokens are converted into lowercase letters.

The main objective in implementing the stop word elimination process is reducing the number of words in a report, which affects NLP speed and performance. In NLP, stop words are words that are ignored in processing, which are usually stored in stop lists. Eliminating stop words in this study is aimed at increasing the similarity between two series of words based on their true meanings.

The following process is known as lemmatization, which is the process of turning tokens into essential words. Lemmatization aims to increase accuracy in the next process and was assisted by Stanford NLP. Calculation using cosine similarity $[29,31]$ is the last process of calculating between-word similarity. Cosine similarity, as implemented in this study, consists of three stages: vector making, calculation of word similarity, and calculation of the cosine values. Each word will be counted as a similarity to other words to obtain words with different meanings. If several similar words are found, only the first word will be included in the vector. Determining words with different meanings requires that the similarity value of the word does not exceed a certain threshold. The similarity of words is calculated using the $\mathrm{Wu}$ and Palmer Semantic Similarity Measure [32], which uses WordNet [33] to calculate the depth.

\subsection{Class diagram semantic similarity}

Before measuring the similarity between two class diagrams, we first divided class diagram elements into property information and relationship information [28]. Fig. 2 shows class diagram elements. Property information consists of class names, attributes, and operations, and relationship information includes association, dependency, and generalization.

Furthermore, Fig. 2 shows that the class diagram semantic similarity (classSem) consists of the similarity of class diagram properties (propSim) and relationships (relSim). Fig. 3 shows the flow of semantic similarity calculations. Both class diagrams will be processed in XML metadata interchange format using a tool. Each diagram is extracted based on class diagram components, and each extraction 


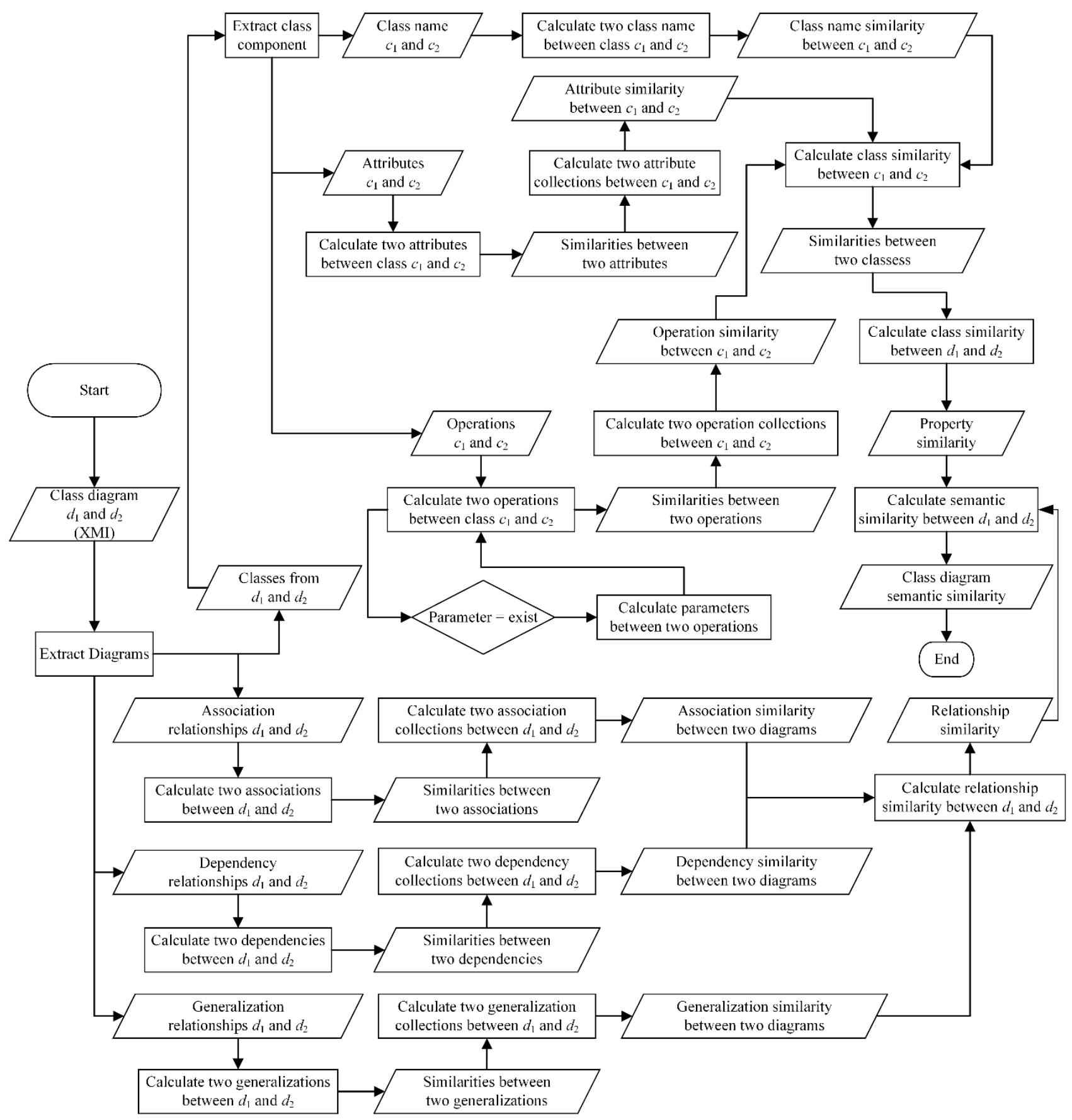

Figure. 3 Flowchart of semantic similarity assessment

result is counted as a semantic similarity. Components are calculated from the smallest to largest. The classSem between class diagrams $d_{1}$ and $d_{2}$ can be expressed as Equation 1 .

$$
\begin{array}{r}
\text { classSem }\left(d_{1}, d_{2}\right)=\left(1-\rho_{\text {sem }}\right) \times \\
\operatorname{propSim}\left(d_{1}, d_{2}\right)+\rho_{\text {sem }} \times \operatorname{relSim}\left(d_{1}, d_{2}\right)(1)
\end{array}
$$

Eq. (1) shows how to assess similarities between class diagrams $d_{1}$ and $d_{2}$. Properties and relationships have a different level of interest using $\rho_{\text {sem }}$, the value of which ranges from 0 to 1 . Then, properties in class diagram $d_{1}$ consist of several classes. Each class in class diagram $d_{1}$ will be compared with the classes in class diagram $d_{2}$. The propSim between the two class diagrams is expressed as Eq. (2).

Class collection $\mathrm{C} 1$ in class diagram $\mathrm{d} 1$ will be compared with class collection $\mathrm{C} 2$ in class diagram $\mathrm{d} 2$. The ith-class (ci) in class diagram d1 is then compared with the jth-class ( $\mathrm{cj}$ ) in class diagram $\mathrm{d} 2$. The similarity between the two classes is called cSim. We used changePivot to change each value in the row and column with a maximum value to 0 . Algorithm 1 explains changePivot. 


$$
\begin{gathered}
\operatorname{propSim}\left(d_{1}, d_{2}\right)=\frac{2 \times\left(\sum_{k=1}^{\operatorname{Min}\left(\left|C_{1}\right|,\left|C_{2}\right|\right)} \operatorname{changePivot}\left(\operatorname{Max}\left(\sum_{i=1}^{\left|C_{1}\right|} \sum_{j=1}^{\left|C_{2}\right|} \operatorname{cSim}\left(c_{i}, c_{j}\right)\right)\right)\right)}{\left|C_{1}\right|+\left|C_{2}\right|} . \\
\operatorname{aSim}\left(c_{1}, c_{2}\right)=\frac{2 \times\left(\sum_{k=1}^{\operatorname{Min}\left(\left|A_{1}\right|,\left|A_{2}\right|\right)} \operatorname{changePivot}\left(\operatorname{Max}\left(\sum_{i=1}^{\left|A_{1}\right|} \sum_{j=1}^{\left|A_{2}\right|} \operatorname{daSim}\left(a_{i}, a_{j}\right)\right)\right)\right)}{\left|A_{1}\right|+\left|A_{2}\right|} . \\
\operatorname{opSim}\left(c_{1}, c_{2}\right)=\frac{2 \times\left(\sum_{k=1}^{\operatorname{Min}\left(\left|O P_{1}\right|,\left|O P_{2}\right|\right)} \operatorname{changePivot}\left(\operatorname{Max}\left(\sum_{i=1}^{\left|O P_{1}\right|} \sum_{j=1}^{\left|O P_{2}\right|} \operatorname{dopSim}\left(o p_{i}, O p_{j}\right)\right)\right)\right)}{\left|O P_{1}\right|+\left|O P_{2}\right|} .
\end{gathered}
$$

\begin{tabular}{l}
\hline Algorithm 1: changePivot \\
\hline Input: two-dimension matrix and \\
pivot/coordinate maximum value $(\mathrm{x}, \mathrm{y})$ \\
1. Select pivot \\
2. $\mathrm{M}(\mathrm{x},:)=0$ \\
3. $\mathrm{M}(:, \mathrm{y})=0$ \\
Output: changed matrix
\end{tabular}

Line 1 in Algorithm 1 takes the pivot or coordinates with the maximum value in the similarity matrix. Line 2 changes all the values in the row pivot (x) to 0 . Line 3 changes all the values in the column pivot (y) to 0 . The cSim between classes $\mathrm{c} 1$ and $\mathrm{c} 2$ can be expressed as Eq. (3)

$$
\begin{aligned}
& \operatorname{cSim}\left(c_{1}, c_{2}\right)=w_{c n} \times \operatorname{cnSim}\left(c_{1}, c_{2}\right)+w_{a} \times \\
& \operatorname{aSim}\left(c_{1}, c_{2}\right)+w_{o p} \times \operatorname{opSim}\left(c_{1}, c_{2}\right) .
\end{aligned}
$$

The similarity between the two classes is influenced by class name similarity ( $\mathrm{cnSim}$ ), attribute similarity (aSim), and operation similarity (opSim). Weight $w_{c n}$ is the weight of $c n$ Sim between classes $c_{1}$ and $c_{2}$. Weight $w_{a p}$ is the weight of aSim between classes $c_{1}$ and $c_{2}$. Weight $w_{o}$ is the weight of opSim between classes $c_{1}$ and $c_{2}$. The total of these weights is 1 . The weight composition was adopted from AlKhiaty [14], which showed that $w_{a}$ and $w_{o p}$ were only slightly greater than $w_{c n}$. Therefore, we used weight compositions $w_{c n}=0.4, w_{a}=0.3$, and $w_{o p}=0.3$. The cnSim value measures the lexical similarity of class names of two classes with semantic similarity described in Section 2.1. Eq. (4) shows aSim between classes $c_{1}$ and $c_{2}$.

We then compared attribute collection $A_{1}$ from class $\mathrm{c}_{1}$ with attribute collection $A_{2}$ from class $c_{1}$. We then used changePivot to change the values of rows and columns in the similarity matrix following Algorithm 1. Attributes then consist of a modifier, an attribute name, and a data type. We must perform a detailed attribute similarity calculation (daSim), which contains these three items. The daSim between attributes $a_{1}$ and $a_{2}$ can be expressed as Eq. (5).

$$
\begin{array}{r}
\operatorname{daSim}\left(a_{1}, a_{2}\right)=w_{m d} \times \operatorname{sim}\left(m d_{1}, m d_{2}\right)+ \\
w_{n a} \times \operatorname{CosineSim}\left(n a_{1}, n a_{2}\right)+w_{t y} \times \\
\operatorname{sim}\left(t y_{1}, t y_{2}\right) .(5)
\end{array}
$$

Weight $w_{m d}$ is the weight of similarity between modifiers $m d_{1}$ and $m d_{2}$. Weight $w_{n a}$ is the weight of attribute name similarity between attribute names $n a_{1}$ and $n a_{2}$. Weight $w_{t y}$ is the weight of data type similarity between data types $t y_{1}$ and $t y_{2}$. The total of $w_{m d}, w_{n a}$, and $w_{t y}$ is 1 , and the weights used are $w_{m d}=$ $0.1, w_{n a}=0.7$, and $w_{t y}=0.2$. We emphasized name attributes because experts see them more clearly. Calculations of similarities between two modifiers and between two data types are simple. If the modifiers of the data types are the same, the similarity value is 1 . If the modifiers or data types are different, the similarity value is 0 . The similarity between attribute names can be calculated from the lexical information of each attribute using semantic similarity, as shown in Section 2.1.

The opSim between all operations in $c_{1}$ and all operations in $c_{2}$ is expressed as Eq. (6). Operation collection $O P_{1}$ in class $c_{1}$ is compared with operation collection $\mathrm{OP}_{2}$ in class $c_{2}$. We then calculated the similarity of each $i$-th operation $\left(o p_{i}\right)$ of class $c_{1}$ with that of the $j$-operation $\left(o p_{j}\right)$ of class $c_{2}$. We then used changePivot to change the values of rows and columns in the similarity matrix following Algorithm 1. Operations consist of a modifier, an operation name, a data type, and a parameter. Therefore, we needed a detailed operation similarity (dopSim) between operations $o p_{1}$ and $o p_{2}$, which contains these four things. dopSim between operations $o p_{1}$ and $o p_{2}$ can be expressed as Eq. (7).

$$
\begin{gathered}
\operatorname{dopSim}\left(\mathrm{op}_{1}, o p_{2}\right)=w_{\text {mdo }} \times \\
\operatorname{sim}\left(\mathrm{mdO}_{1}, \mathrm{mdO}_{2}\right)+w_{\text {nao }} \times \\
\operatorname{CosineSim}\left(\text { naO }_{1}, n a O_{2}\right)+w_{\text {tyo }} \times \\
\operatorname{sim}\left(\text { ty }_{1}, \operatorname{tyO}_{2}\right)+w_{\text {paro }} \times \operatorname{prSim}\left(o p_{1}, o p_{2}\right) .
\end{gathered}
$$

Weight $w_{m d o}$ is the weight of operation modifier similarity between operation modifiers $m d O_{1}$ and $m d O_{2}$. Weight $w_{n a O}$ is the weight of operation name similarity between operation names $\mathrm{naO}_{1}$ and $\mathrm{naO}_{2}$. 


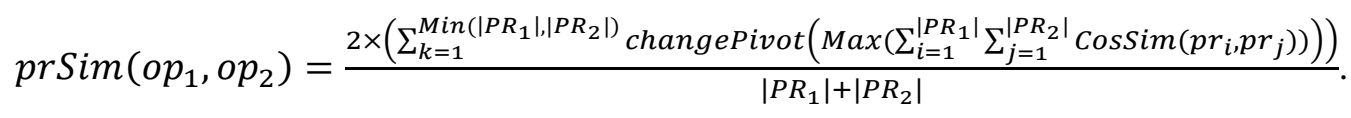

$$
\begin{aligned}
& \operatorname{raSim}\left(d_{1}, d_{2}\right)=\frac{2 \times\left(\sum_{k=1}^{\operatorname{Min}\left(\left|R A_{1}\right|,\left|R A_{2}\right|\right)} \operatorname{changePivot}\left(\operatorname{Max}\left(\sum_{i=1}^{\left|R A_{1}\right|} \sum_{j=1}^{\left|R A_{2}\right|} \operatorname{draSim}\left(r a_{i}, r a_{j}\right)\right)\right)\right)}{\left|R A_{1}\right|+\left|R A_{2}\right|} .
\end{aligned}
$$

Weight $w_{t y o}$ is the weight of operation data type similarity between operation data types tyo $o_{1}$ and $t y O_{2}$. Weight $w_{\text {paro }}$ is the weight of parameter similarity between operations $o p_{1}$ and $o p_{2}$. We used arbitrary weights of $w_{\text {md }}=0.1, w_{\text {naO }}=0.7, w_{t y}=0.1$, and $w_{\text {parO }}$ $=0.1$, and emphasized the name of the operation. Calculation of similarity between modifiers and data types in operations is the same as that between modifiers and data types in attributes. The similarity between operation names can be calculated from the lexical information of each operation using semantic similarity, as shown in Section 2.1. We cannot directly calculate the similarity of parameters ( $\mathrm{prSim}$ ) because an operation can have more than one parameter. prSim is expressed as Eq. (8).

Parameter collection $P R_{1}$ from operation $o p_{1}$ is compared with parameter collection $P R_{2}$ from operation $o p_{2}$. The ith-parameter $\left(p r_{i}\right)$ of the first operation is calculated by the $j t h$-parameter $\left(p r_{j}\right)$ of the second operation. We then used changePivot to change the values of rows and columns in the similarity matrix following Algorithm 1. Similarity between $p r_{i}$ and $p r_{j}$ is calculated using semantic similarity, as shown in Section 2.1. The relSim between class diagrams $d_{1}$ and $d_{2}$ can be expressed as Eq. (9).

$$
\begin{gathered}
r e l \operatorname{Sim}\left(d_{1}, d_{2}\right)=w_{r a} \times \operatorname{raSim}\left(d_{1}, d_{2}\right)+ \\
w_{r d} \times \operatorname{rdSim}\left(d_{1}, d_{2}\right)+w_{r g} \times \operatorname{rgSim}\left(d_{1}, d_{2}\right) .
\end{gathered}
$$

Here, $w_{r a}$ is the weight of association relationship similarity ( $\mathrm{raSim}$ ) between class diagrams $d_{1}$ and $d_{2}$, $w_{r d}$ is the weight of dependency relationship similarity ( $\mathrm{rdSim}$ ) between class diagrams $d_{1}$ and $d_{2}$, and $w_{r g}$ is the weight of generalization relationship similarity ( $\mathrm{rgSim}$ ) between class diagrams $d_{1}$ and $d_{2}$. Relationships are separated because it is unfair to equally compare relationships when the relationships being compared are different. The weight determination is adopted from previous studies [34] by looking at the availability of elements to be calculated. If neither diagram has generalizations, the weight for generalizations is 0 . The weight value to be calculated is the result of sharing the group of relationships sought by the sum relationship of the two diagrams. For example, $d_{1}$ has three associations and one dependency, and $d_{2}$ has two associations.
Thus, the weight of the association is $5 / 6$, the weight of dependency is $1 / 6$, and the weight of generalization is 0 . The raSim value between class diagrams $d_{1}$ and $d_{2}$ can be expressed as Eq. (10).

The association relationship collection $R A_{1}$ from class diagram $d_{1}$ is compared with the association relationship collection $R A_{2}$ from class diagram $d_{2}$. The $i$ th-association $\left(r a_{i}\right)$ from class diagram $d_{1}$ is calculated along with the $j$ th-association $\left(r a_{j}\right)$ from class diagram $d_{2}$, and changePivot is used to change the row and column values in the similarity matrix following Algorithm 1. Furthermore, an association relationship consists of the source class, relation name, aggregation/composition, and target class. Therefore, we need a detailed association relationship similarity ( $\mathrm{raSim})$ between association relationships $r a_{1}$ and $r a_{2}$, which can be expressed as Eq. (11).

$$
\begin{gathered}
\operatorname{draSim}\left(r a_{1}, r a_{2}\right)=w_{r a 1} \times \operatorname{CosSim}\left(\operatorname{src}_{1}, \operatorname{src}_{2}\right) \\
+w_{r a 2} \times \operatorname{CosSim}\left(n m_{1}, n m_{2}\right) \\
+w_{r a 3} \times \operatorname{sim}\left(o w_{1}, o w_{2}\right) \\
+w_{r a 4} \times \operatorname{CosSim}\left(\operatorname{tgt}_{1}, \operatorname{tgt}_{2}\right) .
\end{gathered}
$$

draSim consists of source class $s r c$, relationship name $\mathrm{nm}$, relation ownership ow, and target class tgt. Here, $w_{r a 1}$ is the weight of source class similarity between source classes $s r c_{1}$ and $s r c_{2}, w_{r a 2}$ is the weight of relationship name similarity between relationship names $n m_{1}$ and $n m_{2}, w_{r a 3}$ is the weight of ownership similarity between ownerships $o w_{1}$ and $o w_{2}$, and $w_{r a 4}$ is the weight of target class similarity between target classes $\operatorname{tg} t_{1}$ and $\operatorname{tgt}_{2}$. The total weight is 1 , and the weights used are $w_{r a 1}=0.3, w_{r a 2}=0.2$, $w_{r a 3}=0.2$, and $w_{r a 4}=0.3$. The weight of the class name (source class and target class) is determined to be greater than that of the ownership and relationship name because they are always available in class diagrams. In contrast, relationship names are rarely written on class diagrams and ownership consists only of composition or aggregation. Class name and relationship name similarities use semantic similarity, as shown in Section 2.1. The value of ownership similarity is 0 if they are different and 1 if they are not different. The $r d S i m$ between class diagrams $d_{1}$ and $d_{2}$ can be expressed as Eq. (12).

Here, dependency relationship collection $R D_{1}$ from class diagram $d_{1}$ is compared with dependency relationship collection $R D_{2}$ from class diagram $d_{2}$. 


$$
\begin{gathered}
\operatorname{rdSim}\left(d_{1}, d_{2}\right)=\frac{2 \times\left(\sum_{k=1}^{\operatorname{Min}\left(\left|R D_{1}\right|,\left|R D_{2}\right|\right)} \operatorname{changePivot}\left(\operatorname{Max}\left(\sum_{i=1}^{\left|R D_{1}\right|} \sum_{j=1}^{\left|R D_{2}\right|} \operatorname{drdSim}\left(r d_{i}, r d_{j}\right)\right)\right)\right)}{\left|R D_{1}\right|+\left|R D_{2}\right|} . \\
\operatorname{drdSim}\left(r d_{1}, r d_{2}\right)=\frac{\operatorname{CosSim}\left(\operatorname{src}_{1}, \operatorname{src} c_{2}\right)+\operatorname{CosSim}\left(\operatorname{tgt}_{1}, t g t_{2}\right)}{2} . \\
\operatorname{rgSim}\left(d_{1}, d_{2}\right)=\frac{2 \times\left(\sum_{k=1}^{\operatorname{Min}\left(\left|R G_{1}\right|,\left|R G_{2}\right|\right)} \operatorname{changePivot}\left(\operatorname{Max}\left(\sum_{i=1}^{\left|R G_{1}\right|} \sum_{j=1}^{\left|R G_{2}\right|} \operatorname{drgSim}\left(r g_{i}, r g_{j}\right)\right)\right)\right)}{\left|R G_{1}\right|+\left|R G_{2}\right|} . \\
\operatorname{drgSim}\left(r g_{1}, r g_{2}\right)=\frac{\operatorname{CosSim}\left(\operatorname{src}_{1}, \operatorname{src}_{2}\right)+\operatorname{CosSim}\left(\operatorname{tgt}_{1}, \operatorname{tgt}_{2}\right)}{2} .
\end{gathered}
$$

The $i$ th-dependency relationship $\left(r d_{i}\right)$ from class diagram $d_{1}$ is calculated along with the $j$ thdependency relationship $\left(r d_{j}\right)$ from class diagram $d_{2}$, and changePivot is used to change the row and column values in the similarity matrix following Algorithm 1. The dependency relationship consists of the source and target classes. Therefore, we need an advanced dependency relationship similarity (drdSim) between dependency relationships $r d_{1}$ and $r d_{2}$, which can be expressed as Eq. (13).

Class name (source class and target class) similarity uses semantic similarity as shown in Section 2.1. The generalization relationship similarity ( $\mathrm{rgSim}$ ) between class diagrams $d_{1}$ and $d_{2}$ can be expressed as Eq. (14).

Generalization relationship collection $R G_{1}$ from class diagram $d_{1}$ is compared with generalization relationship collection $R G_{2}$ from class diagram $d_{2}$. The $i$ th-generalization relationship $\left(r g_{i}\right)$ from class diagram $d_{1}$ is calculated along with the $j$ thgeneralization relationship $\left(r g_{j}\right)$ from class diagram $d_{2}$, and changePivot is used to change the row and column values in the similarity matrix following Algorithm 1. Furthermore, the generalization consists of the source and target classes. Therefore, we need an advanced generalization relationship similarity ( $\mathrm{drgSim})$ between generalization relationships $r g_{1}$ and $r g_{2}$, which can be expressed as Eq. (15).

The class name similarity includes the source $(s r c)$ and target class names (tgt), and class name similarity is calculated along with semantic similarity, as shown in Section 2.1.

\section{Structural similarity assessment}

Structural similarity in class diagrams can be represented by making a UML common graph (UCG) model of each diagram. Thus, the pair of diagrams for which similarity will be calculated is converted to UCG, and the resulting UCG pair is searched for similarity values.

\subsection{Class diagram structural similarity}

UCG models were originally intended to build graphs that can accommodate several UML diagrams with the same characters. In this study, we proposed a graph that can provide both class and sequence diagrams. The concept is that the UCG model can calculate structural similarity between two class diagrams and between sequence diagrams, as well as the similarity of class and sequence diagrams. UCG models are currently used to calculate the structural similarity between two class diagrams. As with graphs in general, UCG consists of two elements, namely, vertices and edges. The UCG used in this study is thus an improvement on conventional UCG [21].

A UCG can only be used to create models for class diagrams. In this study, we built a model that can include several UML diagrams. Table 1 shows the designation of the UCG model, which provides for class and sequence diagrams. To this, we added object vertex, class edge, reply message edge, synchronous message edge, and asynchronous message edge components. We propose calculations on sequence diagrams similar to class diagram

Table 1. UML common graph description

\begin{tabular}{|l|l|l|l|}
\hline No & Type & Name & Tag \\
\hline 1 & Vertex & Class Vertex & vc \\
\hline 2 & Vertex & Attribute Vertex & va \\
\hline 3 & Vertex & Operation Vertex & vo \\
\hline 4 & Vertex & Parameter Vertex & vp \\
\hline 5 & Vertex & Object Vertex & vob \\
\hline 6 & Edge & Attribute Edge & $\mathrm{e}_{\mathbf{a}}$ \\
\hline 7 & Edge & Operation Edge & $\mathrm{e}_{\mathrm{o}}$ \\
\hline 8 & Edge & Parameter Edge & $\mathrm{e}_{\mathrm{p}}$ \\
\hline 9 & Edge & Association Edge & $\mathrm{e}_{1}$ \\
\hline 10 & Edge & Generalization Edge & $\mathrm{e}_{2}$ \\
\hline 11 & Edge & Aggregation Edge & $\mathrm{e}_{3}$ \\
\hline 12 & Edge & Composition Edge & $\mathrm{e}_{4}$ \\
\hline 13 & Edge & Dependency Edge & $\mathrm{e}_{5}$ \\
\hline 14 & Edge & Realization Edge & $\mathrm{e}_{6}$ \\
\hline 15 & Edge & Class Edge & $\mathbf{e}_{\mathbf{c}}$ \\
\hline 16 & Edge & Reply Message Edge & $\mathbf{e}_{7}$ \\
\hline 17 & Edge & Synchronous Message Edge & $\mathbf{e}_{\mathbf{8}}$ \\
\hline 18 & Edge & Asynchronous Message Edge & $\mathbf{e}_{\mathbf{9}}$ \\
\hline
\end{tabular}




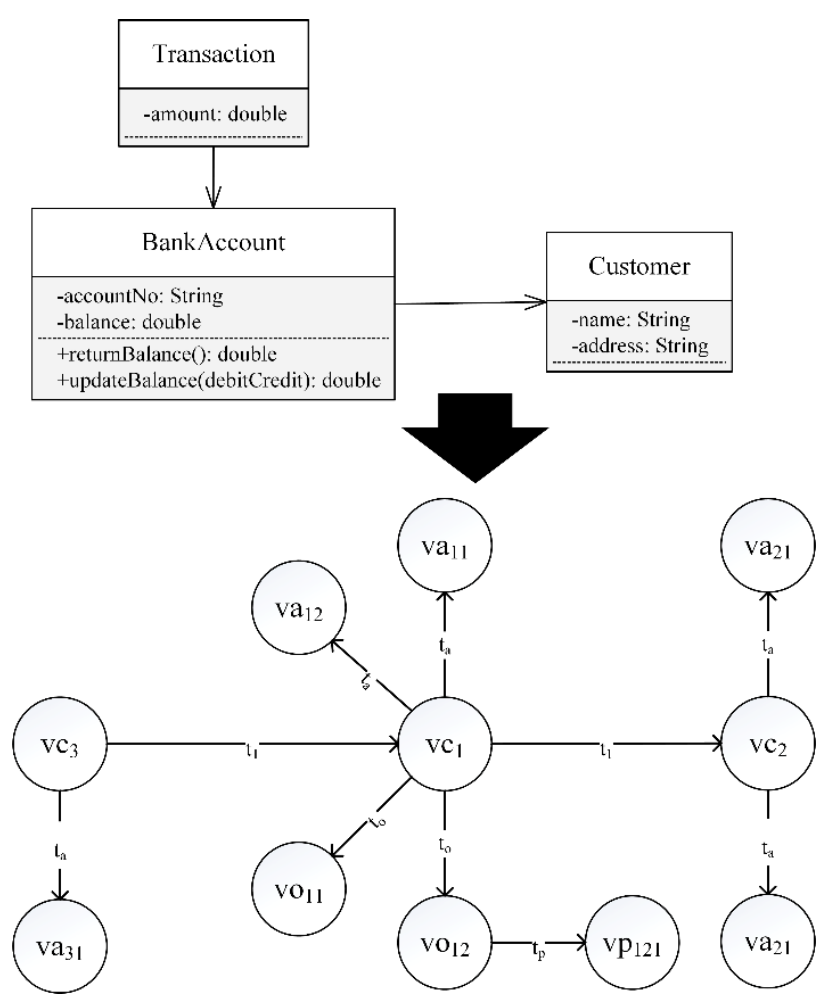

Figure. 4 Transformation of a class diagram into a UML common graph

calculations. These calculations are based on interactions between classes in the class diagram that also occur in the sequence diagram (intraSim). The difference is the information in the class (interSim).

Following Table 1, we can transform a class diagram to a UCG. Fig. 4 illustrates this process. The graph shows that a class can have zero or more attributes, zero or more operations, and zero or more related classes. Thus, an operation can also have zero or more parameters.

\subsection{UCG similarity assessment}

We obtained the structural similarity assessment of the class diagram from the UCG similarity assessment. Fig. 5 shows an assessment of structural similarity. Diagrams are divided based on structure and then calculated from the smallest graph. The results of these calculations are then entered into the next calculation.

All proposed structural calculations disregard the lexical information of the class diagram because structural similarity is different from semantic similarity, as explained previously. Structural similarity only considers the types of components in the class diagram. The UCG of class diagrams contains two types of information: intraStructure and interStructure [21]. IntraStructure (intraSim) contains the number of attributes and operations of

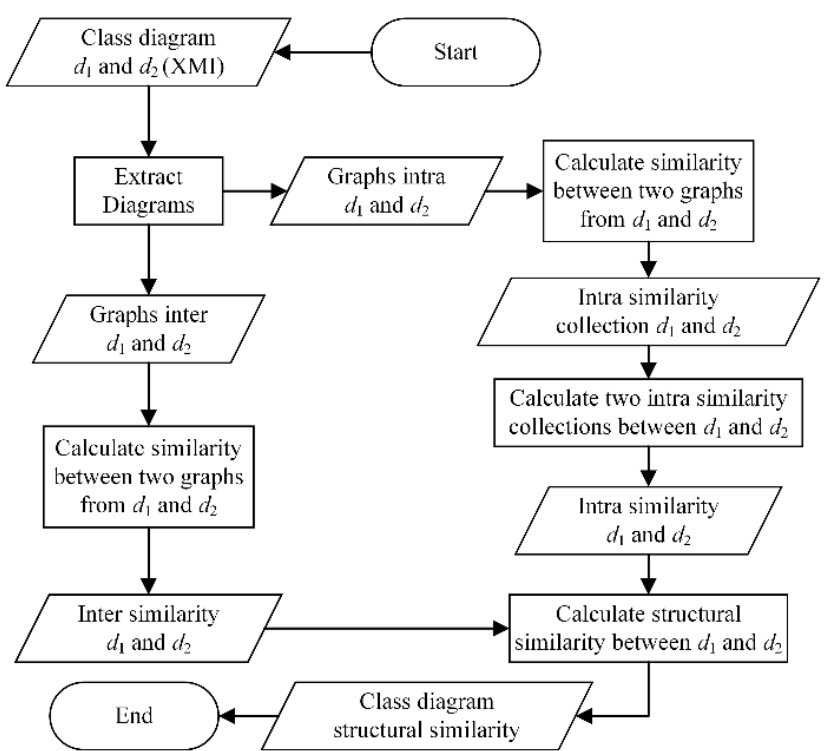

Figure. 5 Flowchart of structural similarity assessment

each class, and interStructure (interSim) includes the relationship information between the two classes. Consequently, the structural similarity between two UCGs must consider both classes of information, the importance of which is distinguished by $\rho_{\text {struc }}$, which ranges from 0 to 1 . Eq. (16) shows the structural assessment between class diagrams $d_{1}$ and $d_{2}$.

$$
\begin{array}{r}
\operatorname{classStruc}\left(d_{1}, d_{2}\right)=\left(1-\rho_{\text {struc }}\right) \times \\
\operatorname{intraSim}\left(g_{1}, g_{2}\right)+\rho_{\text {struc }} \times \operatorname{interSim}\left(g_{1}, g_{2}\right) .
\end{array}
$$

Graphs $g_{1}$ and $g_{2}$ are the initial and the comparative UCGs, respectively. Unlike previous studies, we calculated intraSim by combining GED [35] using greedy algorithms. Eq. (17) shows how to calculate intraSim. The information in each class contains numbers of attributes, operations, and parameters. Thus, a graph is split into several subgraphs based on classes. Here, $v c_{i}$ is a subgraph of $g_{1}$ and $g_{2}$. Thus, each class of $g_{1}$ is calculated using the GED for all classes of $g_{2}$ as Eq. (17).

Here, $V C_{1}$ is a collection of subgraphs in $g_{1}$, and $V C_{2}$ is a collection of subgraphs in $g_{2}$. The changePivot function is to change the all points row and column values to 0 following Algorithm 1. After determining the similarity of the two subgraphs between $g_{1}$ and $g_{2}$ using GED (GED ( $\left.v c i, v c j\right)$ ), the greedy algorithm finds the optimal similarity value between the two. This value is then assigned to be the value of intraSim. GED calculates the minimum cost to convert $g_{1}$ to $g_{2}$, and the minimum costs are calculated as Eq. (18). 


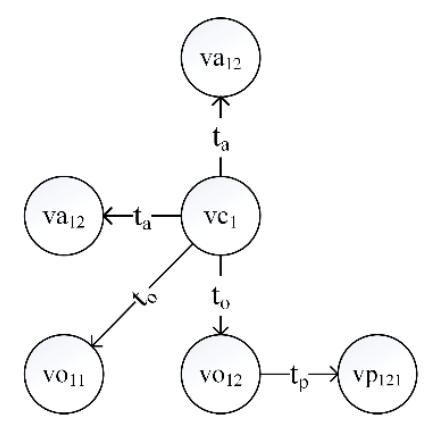

$\left(g_{1}\right)$

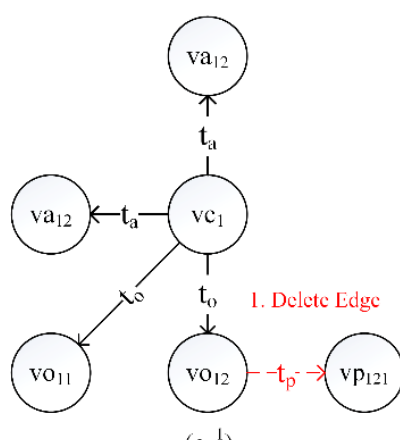

$\left(g_{1}{ }^{1}\right)$
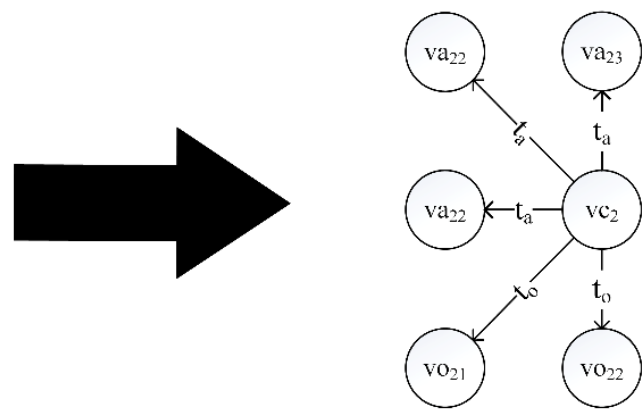

$\left(g_{2}\right)$

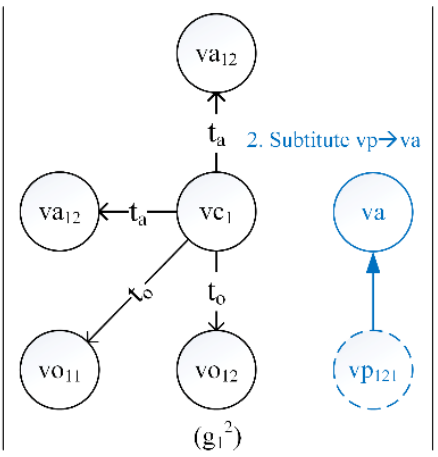

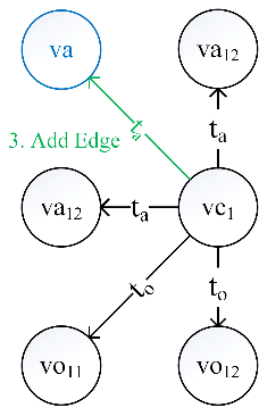

$\left(\mathrm{g}_{1}{ }^{3}\right)$

Figure. 6 Subgraph matching using graph edit distance in intraSim

$$
\operatorname{intraSim}\left(g_{1}, g_{2}\right)=\frac{2 \times\left(\sum_{k=1}^{\operatorname{Min}\left(\left|V C_{1}\right|,\left|V C_{2}\right|\right)} \operatorname{changePivot}\left(\operatorname{Max}\left(\sum_{i=1}^{\left|V C_{1}\right|} \sum_{j=1}^{\left|V C_{2}\right|} G E D\left(v c_{i}, v c_{j}\right)\right)\right)\right)}{\left|V C_{1}\right|+\left|V C_{2}\right|} .
$$

$$
d_{\lambda_{\min }}\left(g_{1}, g_{2}\right)=\min _{\lambda \in \gamma\left(g_{1}, g_{2}\right)} \sum_{e_{i} \in \lambda} c\left(e_{i}\right)
$$

Here, $d_{\lambda_{\min }}$ is the lowest cost graph path. GED searches for all possible permutations of the path and calculates the cost of changing $g_{1}$ to $g_{2}$. The GED we used has three operations: substitution, addition, and deletion. Substitution is accomplished by changing the edges, addition is done by adding edges and vertices according to the destination graph, and deletion is performed by removing edges and vertices according to the destination graph. Here, $\lambda_{\text {min }}$ produces the smallest change cost amount, so it must be normalized into a $0-1$ scale. Fig. 6 illustrates how $g_{1}$ subgraphs are changed into $g_{2}$ subgraphs to calculate intraSim. Fig. 6 shows that the minimum cost for converting the first subgraph into the second subgraph is 3 . Therefore, the normalized value of similarity for the two subgraphs is $1-(3 / 11) .11$ is obtained by taking the maximum of the sum of vertices and edges in $g_{1}$ and $g_{2}$.

Unlike previous studies, we used GED [36] to measure interSim, which only takes the relationship information between classes from UCGs, meaning that the shape of the graph to be calculated differs from intraSim. Fig. 7 shows an example of the form of interSim used to measure the similarity of two class diagrams, $\mathrm{CD}_{1}$ and $\mathrm{CD}_{2}$. $\mathrm{CD}_{1}$ has three classes and two relations, and $\mathrm{CD}_{2}$ has three classes and three relations.

Prior research used a maximum common subgraph that was more suitable for reuse. In this study, we used GED because it matches the similarity assessment that must not see a portion of the graph content. Eq. (19) measures interSim with the concept of GED.

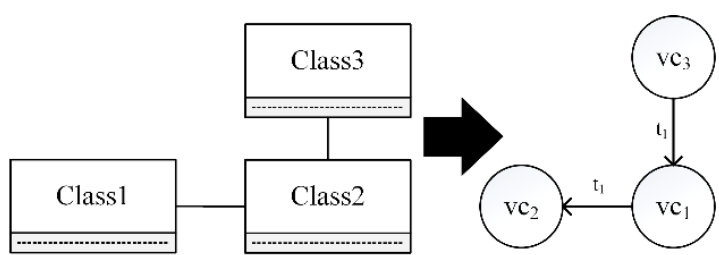

(CD1)

$\left(g_{1}\right)$

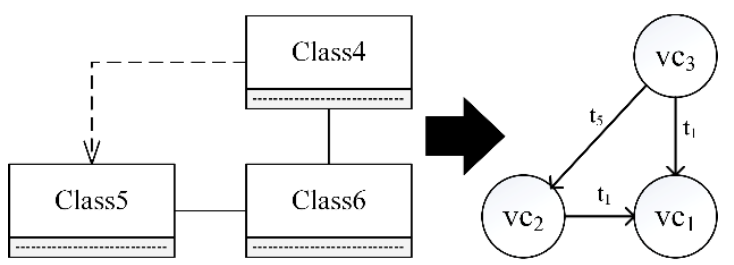

$\left(\mathrm{g}_{2}\right)$

Figure. 7 Graph used to measure interSim 


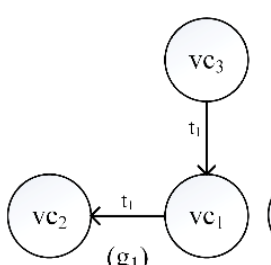

$\left(g_{1}\right)$

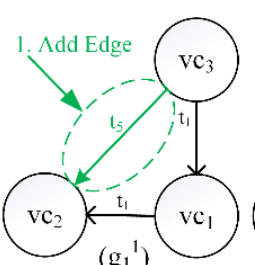

$\left(g_{1}{ }^{1}\right)$

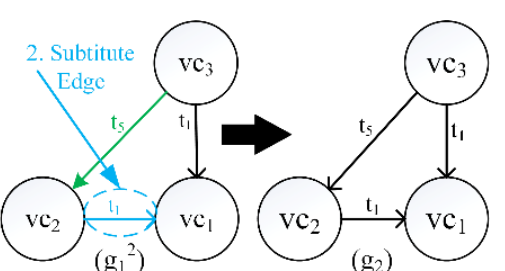

$\left(\mathrm{g}_{2}\right)$

Figure. 8 Graph matching using graph edit distance in interSim

\begin{tabular}{|c|c|}
\hline Variable & Description \\
\hline classSem & Semantic assessment \\
\hline$d_{1}$ & Firts class diagram \\
\hline$d_{2}$ & Second class diagram \\
\hline$\rho_{\text {sem }}$ & $\begin{array}{l}\text { Distinguishing semantic } \\
\text { component importance }\end{array}$ \\
\hline propSim & Property similarity \\
\hline relSim & Relationship similarity \\
\hline$C_{1}$ & Class collection of $d_{1}$ \\
\hline$C_{2}$ & Class collection of $d_{2}$ \\
\hline$c_{i}$ & The $i$-class from $C_{1}$ \\
\hline$c_{j}$ & The $j$-class from $C_{2}$ \\
\hline$c \operatorname{Sim}$ & Class similarity \\
\hline cnSim & Class Name Similarity \\
\hline aSim & Attribute Similarity \\
\hline opSim & Operation Similarity \\
\hline$w_{c n}$ & $\begin{array}{l}\text { Weight of class name } \\
\text { similarity }\end{array}$ \\
\hline$w_{a}$ & $\begin{array}{l}\text { Weight of attribute } \\
\text { similarity }\end{array}$ \\
\hline$w_{o p}$ & $\begin{array}{l}\text { Weight of operation } \\
\text { similarity }\end{array}$ \\
\hline$A_{1}$ & Attribute collection of $c_{1}$ \\
\hline$A_{2}$ & Attribute collection of $c_{2}$ \\
\hline$a_{i}$ & The $i$-attribute from $A_{1}$ \\
\hline$a_{j}$ & The $j$-attribute from $A_{2}$ \\
\hline daSim & $\begin{array}{l}\text { Detailed attribute } \\
\text { similarity }\end{array}$ \\
\hline$m d_{1}$ & Modifier of attribute $a_{1}$ \\
\hline$m d_{2}$ & Modifier of attribute $a_{2}$ \\
\hline$n a_{1}$ & Name of attribute $a_{1}$ \\
\hline$n a_{2}$ & Name of attribute $a_{2}$ \\
\hline$t y_{1}$ & Data type of attribute $a_{1}$ \\
\hline$t y_{2}$ & Data type of attribute $a_{2}$ \\
\hline$w_{m d}$ & Weight of modifier \\
\hline$w_{n a}$ & Weight of attribute name \\
\hline$w_{t y}$ & Weight of data type \\
\hline$O P_{1}$ & Operation collection of $c$ \\
\hline
\end{tabular}

Table 2. Variable description

\begin{tabular}{|c|c|}
\hline Variable & Description \\
\hline $\mathrm{OP}_{2}$ & Operation collection of $c_{2}$ \\
\hline$o p_{i}$ & The $i$-operation of $O P_{1}$ \\
\hline$o p_{j}$ & The $j$-operation of $O P_{2}$ \\
\hline $\begin{array}{l}\text { dopSi } \\
m\end{array}$ & $\begin{array}{l}\text { Detailed operation } \\
\text { similarity }\end{array}$ \\
\hline$m d O_{1}$ & Modifier of $o p_{1}$ \\
\hline$m d O_{2}$ & Modifier of $\mathrm{op}_{2}$ \\
\hline$n a O_{1}$ & Name of operation $o p_{1}$ \\
\hline $\mathrm{naO}_{2}$ & Name of operation $o p_{2}$ \\
\hline$t y O_{1}$ & Data type of $o p_{1}$ \\
\hline$t y O_{2}$ & Data type of $\mathrm{op}_{2}$ \\
\hline prSim & Parameter similarity \\
\hline$w_{m d O}$ & $\begin{array}{l}\text { Weight of operation } \\
\text { modifier similarity }\end{array}$ \\
\hline wnaO & $\begin{array}{l}\text { Weight of operation } \\
\text { name similarity }\end{array}$ \\
\hline$w_{t y O}$ & $\begin{array}{l}\text { Weight of operation data } \\
\text { type similarity }\end{array}$ \\
\hline$w_{\text {parO }}$ & $\begin{array}{l}\text { Weight of parameter } \\
\text { similarity }\end{array}$ \\
\hline$P R_{1}$ & $\begin{array}{l}\text { Parameter collection of } \\
\text { operation } o p_{1}\end{array}$ \\
\hline$P R_{2}$ & $\begin{array}{l}\text { Parameter collection of } \\
\text { operation } \mathrm{op}_{2}\end{array}$ \\
\hline$p r_{i}$ & The $i$-parameter of $o p_{1}$ \\
\hline$p r_{j}$ & The $j$-parameter of $o p_{2}$ \\
\hline relSim & Relationship similarity \\
\hline raSim & Association similarity \\
\hline rdSim & Dependency similarity \\
\hline rgSim & Generalization similarity \\
\hline$w_{r a}$ & $\begin{array}{l}\text { Weight of association } \\
\text { similarity }\end{array}$ \\
\hline$w_{r d}$ & $\begin{array}{l}\text { Weight of dependency } \\
\text { similarity }\end{array}$ \\
\hline$w_{r g}$ & $\begin{array}{l}\text { Weight of generalization } \\
\text { similarity }\end{array}$ \\
\hline$R A_{1}$ & $\begin{array}{l}\text { Association collection } \\
\text { from } d_{1}\end{array}$ \\
\hline$R A_{2}$ & $\begin{array}{l}\text { Association collection } \\
\text { from } d_{2}\end{array}$ \\
\hline$r a_{i}$ & The $i$-relationship of $R A_{1}$ \\
\hline$r a_{j}$ & The $j$-relationship of $R A_{2}$ \\
\hline draSim & $\begin{array}{l}\text { Detailed association } \\
\text { similarity }\end{array}$ \\
\hline$s r c$ & Source class name \\
\hline
\end{tabular}

\begin{tabular}{|c|c|}
\hline Variable & Description \\
\hline$n m$ & Name of the relationship \\
\hline ow & aggregation/composition \\
\hline $\operatorname{tg} t$ & Target class name \\
\hline$w r a_{1}$ & Weight of source class name \\
\hline$w_{r a}$ & Weight of relationship name \\
\hline$w_{r a}$ & Weight of relationship own \\
\hline$w_{r a}$ & Weight of target class name \\
\hline$R D_{1}$ & Dependency collection of $d_{1}$ \\
\hline$R D_{2}$ & Dependency collection of $d_{2}$ \\
\hline$r d_{i}$ & The $i$-relationship of $R D_{1}$ \\
\hline$r d_{j}$ & The $j$-relationship of $R D_{2}$ \\
\hline drdSim & $\begin{array}{l}\text { Detailed dependency } \\
\text { similarity }\end{array}$ \\
\hline$R G_{1}$ & $\begin{array}{l}\text { Generalization collection of } \\
d_{1}\end{array}$ \\
\hline$R G_{2}$ & $\begin{array}{l}\text { Generalization collection of } \\
d_{2}\end{array}$ \\
\hline$r g_{i}$ & The $i$-relationship of $R G_{1}$ \\
\hline$r g_{j}$ & The $j$-relationship of $R G_{2}$ \\
\hline drgSim & $\begin{array}{l}\text { Detailed generalization } \\
\text { similarity }\end{array}$ \\
\hline classStruc & Structural assessment \\
\hline$\rho_{\text {struc }}$ & $\begin{array}{l}\text { Distinguishing structural } \\
\text { component importance }\end{array}$ \\
\hline intraSim & Similarity of intraStructure \\
\hline interSim & Similarity of interStructure \\
\hline$g_{1}$ & Translated graph of $d_{1}$ \\
\hline$g_{2}$ & Translated graph of $d_{2}$ \\
\hline$V C 1$ & Collection of subgraphs in $g_{1}$ \\
\hline$V C 2$ & Collection of subgraphs in $g_{1}$ \\
\hline$v c_{i}$ & The $i$-graph from $V C_{1}$ \\
\hline$v c_{j}$ & The $j$-graph from $V C_{2}$ \\
\hline$d_{\lambda_{\text {min }}}$ & The lowest cost graph path \\
\hline$c\left(e_{i}\right)$ & $\begin{array}{l}\text { The cost of transforming } g_{1} \\
\text { into } g_{2}\end{array}$ \\
\hline classSim & $\begin{array}{l}\text { Class diagram similarity } \\
\text { assessment }\end{array}$ \\
\hline$\rho$ & $\begin{array}{l}\text { Distinguishing assessment } \\
\text { component importance }\end{array}$ \\
\hline
\end{tabular}

$$
\operatorname{inter} \operatorname{Sim}\left(g_{1}, g_{2}\right)=1-\frac{d \lambda_{\min }\left(g_{1}, g_{2}\right)}{\max \left(\left|g_{1}\right|,\left|g_{2}\right|\right)}
$$

Here, $d \lambda_{\min }$ is the minimum cost to convert $g_{1}$ into $g_{2}$, and $\max \left(\left|g_{1}\right|,\left|g_{2}\right|\right)$ is the most significant value of the sum of edges and vertices in $g_{1}$ or $g_{2}$. Fig. 8 shows 
graph matching with GED in interSim and shows that 2 is the cost of converting $g_{1}$ to $g_{2}$. There are add vertex and substitute edge operations.

\section{Class diagram assessment}

The proposed class similarity diagram (classSim) assessment combines semantic and structural similarities as a similarity assessment parameter. The similarity of class diagrams $d_{1}$ and $d_{2}$ is determined using Eq. (20). Semantic similarity (classSem) uses all lexical information that appears in the class diagram, and structural similarity (classStruc) uses a graph model representing the class diagrams. Structural and semantic similarities have different importance with respect to $\rho$, the value of which ranges from 0 to 1 .

$$
\begin{gathered}
\text { classSim }=(1-\rho) \times \text { classSem }\left(d_{1}, d_{2}\right)+ \\
\rho \times \operatorname{classStruc}\left(d_{1}, d_{2}\right) .
\end{gathered}
$$

The explanation of each variable from Eq. (1) to 20 can be seen in Table 2 .

\section{Result}

\subsection{Dataset}

The purpose of this study is to develop an automatic assessment method. Therefore, the data are a collection of class diagrams that are answers to student assessments. In the assessments, we gave students three questions related to making class diagrams, and each question contained a different project. Table 3 shows the project details. The projects present information on use case descriptions, and students construct class diagrams based on these use case descriptions. Each project has an answer key that serves as a reference for assessment. The collected class diagram consists of 31 diagrams from three different projects.

\subsection{Gold standard}

A gold standard serves as a reference for evaluating a proposed method's reliability. Thus, the proposed method's reliability refers to its agreement with a gold standard developed by experts. We measured the agreement using Gwet's AC1 [37].

Table 3. Summary of the collected class diagram

\begin{tabular}{|l|l|c|c|c|}
\hline \multicolumn{1}{|c|}{ Project } & Description & Answers & $\begin{array}{l}\text { Class } \\
\text { Average }\end{array}$ & $\begin{array}{c}\text { Relationship } \\
\text { Average }\end{array}$ \\
\hline Outlay & $\begin{array}{l}\text { Financial } \\
\text { recording }\end{array}$ & 11 & 9 & 7 \\
\hline Library & Book rental & 10 & 9 & 9 \\
\hline QuickBill & Point of sale & 10 & 6 & 6 \\
\hline
\end{tabular}

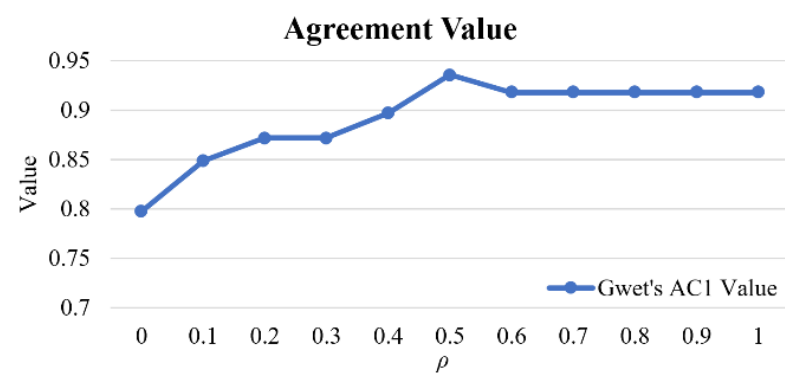

Figure. 9 The maximum agreement value of each $\rho$

The gold standard in this study was built based on expert evaluation questionnaires. Experts were asked to assess student answers to the questions and were given answer keys. The assessment range was 0-100. The experts were software engineering lecturers who have taught class diagrams for two to fourteen years at bachelor degree programs. In this study, we relied on 27 experts who assessed 31 pairs of student and key answers. The nationality of the entire expert is Indonesian. Age of experts ranging from thirty to fifty-two years old. The experts are from nine different universities in Indonesia.

\subsection{Similarity assessment evaluation}

We evaluated similarity assessment by comparing the proposed method's results with those from the gold standard using Gwet's AC1 to measure inter-rater reliability. We normalized expert assessment values and the proposed method's results on a $1-5$ scale, where the scale number represents a range of assessment values with an even distribution $(1:<20,2:<40,3:<60,4:<80$, and 5: $>80)$. The proposed method is assessed using all combinations of $\rho, \rho_{\text {sem }}$, and $\rho_{\text {struc }}$ as differentiators of the importance of assessment components. The experiment was repeated 1331 times with different weight combinations, and the weights were tweaked with the same inputs. The values of $\rho, \rho_{\text {sim }}$, and $\rho_{\text {struc }}$ were $0,0.1$, and 0.2 to 1 , respectively. Fig. 9 shows the maximum agreement value for each instance of $\rho$. The maximum agreement value was 0.935 at $\rho=0.5$, $\rho_{\text {sem }}=0.3$, and $\rho_{\text {struc }}=0$ and 0.1 .

\section{Discussion}

The maximum agreement value resulting from our evaluation indicates that the proposed method can function as reliably as an expert can. This is because experts and the proposed method have an almost perfect level of agreement. We found that experts look at the semantic and structural aspects of class diagrams equally when assessing class diagram similarity. Class diagrams are easy to visually understand using the lexical information they contain. 


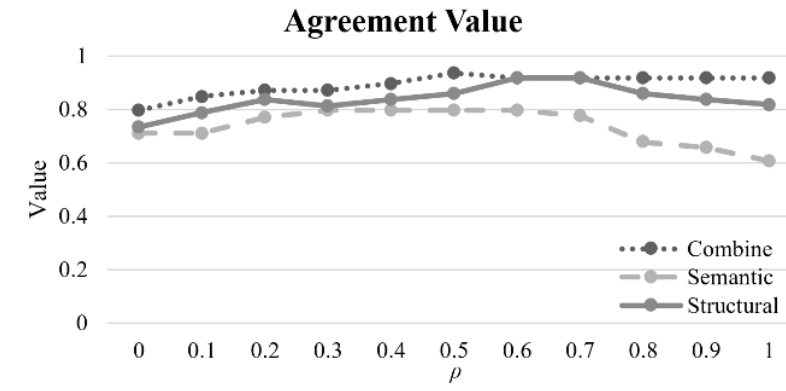

Figure. 10 Comparison of agreement with previous research in assessment case

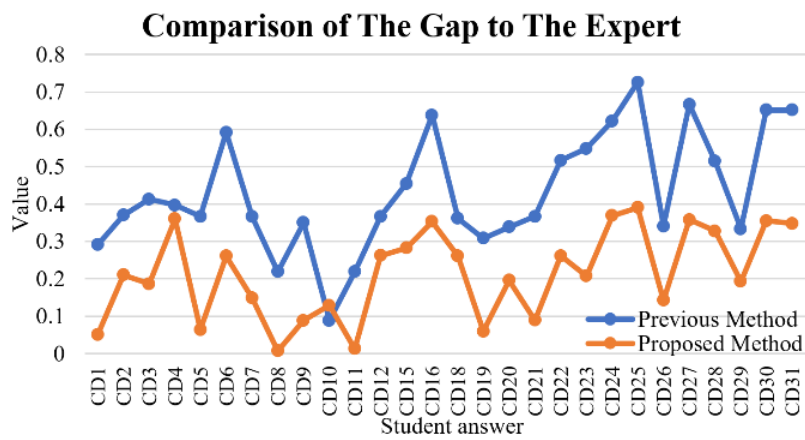

Figure. 11 Comparison of the gap to the experts

For semantic similarity, experts tend to look at the lexical information from the class separately and tend to look at the property information for each class. Experts do not pay more attention to relationships between classes when making an assessment. For structural similarity, experts look at the structure in the class almost exclusively, rather than considering within-class and between-class structures equally.

We conducted several evaluations of expert judgment before using those judgments as a gold standard. First, we tested the collection of expert answers from each pair of diagrams for correlation using the Pearson correlation coefficient [38]. This stage reduces the three pairs of diagrams, eliminating those that do not have strong correlations or have too many different answers. Second, we conducted an inter-rater reliability evaluation [39] to show the level of agreement among the experts. During this stage, we eliminated five experts to provide more reliable and homogeneous answers. The final test result for data reliability was 0.935 on a $0-1$ scale. Therefore, we conclude that the data used re reliable.

We compared research that focused solely on semantic aspects [28] and research that considers only structural aspects [21] with our proposed method that uses both. Fig. 10 shows that using structural and semantic combinations with individual weights can provide better agreement values than separate assessment.

The maximum value of each $\rho$ in semantic similarity tends to be more significant on the left side of the chart. In line with our proposed method, experts tend to look at the properties of each class rather than between-class relationships when assessing semantic aspects alone. In contrast, the maximum value of each $\rho$ in structural similarity tends to be higher on the right side of the chart. Experts tend to look at relationships between classes rather than within-class structures when assessing structural aspects alone, which differ slightly from our proposed method. The structural similarity tendency still cannot be concluded in general because experts have difficulty accurately measuring the similarity of structures between two class diagrams. We attempted small experiments to measure simple class diagrams and found that experts cannot assess structural similarity as accurately as the proposed method. The proposed method uses GED, which can accurately compare all class relationships. Several factors can influence the accuracy gap between expert assessment and that of the proposed method. These factors include the number of classes, the complexity of each class's property structure, and the number of relationships between classes. Experts find assessment increasingly difficult if the number of these factors increases.

We also compared the results of this study with previous studies. Ali [40] assessed the syntactic similarity of class names, attribute names, and operation names. Fig. 11 shows the results of the comparison. We calculate the gap from the output of our method and Ali's method to the expert assessment. The assessment results use a value range of 0 to 1 where zero is not the same, and one is the same. Based on Fig. 11, 27 of the 28 assessments carried out by our method had a lower gap with the expert assessment. Therefore, our proposed method using semantic and structural assessment provides a closer value to the expert.

The class diagrams used are not for entire projects. We use this limitation to maintain the reliability of the gold standard that is built on expert interpretation. Experts have difficulty assessing the similarity of two diagrams if those diagrams have large and complex shapes. Additionally, experts will be inconsistent when assessing many problems with complex class diagrams.

\section{Conclusion}

Our proposed automated assessment method can determine the similarity of two class diagrams as reliably as an expert. The level of agreement between the proposed method and experts was 0.935. This means that our proposed method has an almost perfect agreement with expert assessment. Class 
diagram similarity is divided into semantic similarity and structural similarity. Combining these two similarities with a specific weight enables the best agreement with experts. Semantic similarity is an aspect experts emphasize in the case of similarity assessment.

This research can be developed further. First, our assessment concept can be used with other UML diagrams because every UML diagram has both semantic and structural aspects. Second, further research is needed to ascertain experts' tendency to assess structural similarity to become an assessment standard that would be used in all assessment cases. Each similarity's weight can be examined for purposes other than assessment such as software reuse and clone detection.

\section{Conflicts of Interest}

The authors declare no conflict of interest.

\section{Author Contributions}

Conceptualization, Reza Fauzan and Daniel Siahaan; methodology, Reza Fauzan and Daniel Siahaan; software, Reza Fauzan; validation, Reza Fauzan, Daniel Siahaan, and Siti Rochimah; formal analysis, Reza Fauzan, Daniel Siahaan, and Siti Rochimah; investigation, Evi Triandini; resources, Evi Triandini; data curation, Siti Rochimah; writing - original draft preparation, Reza Fauzan; writing - review and editing, Daniel Siahaan and Siti Rochimah; visualization, Reza Fauzan and Evi Triandini; supervision, Daniel Siahaan and Siti Rochimah; project administration, Daniel Siahaan.

\section{Acknowledgments}

This research was funded by the Ministry of Research and Technology/National Research and Innovation Agency of the Republic of Indonesia. This research is a collaboration amongst Institut Teknologi Sepuluh Nopember, Politeknik Negeri Banjarmasin, and ITB STIKOM Bali.

\section{References}

[1] L. J. Gonçales and K. Farias, "Towards a hybrid approach to measure similarity between UML models", In: Proc. of ACM Int. Conf. Ser., 2019.

[2] L. J. Gonçales, K. Farias, and V. Bischoff, "On the effects of developers' intuition on measuring similarity between UML models", In: Proc. of ACM Int. Conf. Ser., 2019.

[3] W. N. Robinson and H. G. Woo, "Finding reusable UML sequence diagrams automatically", IEEE Softw., Vol. 21, No. 5, pp. 60-67, 2004, doi: 10.1109/MS.2004.1331304.

[4] A. Adamu and W. M. N. W. Zainoon, "A Framework for Enhancing the Retrieval of UML Diagrams", In: Proc. of Software Reuse: Bridging with Social-Awareness 15th International Conference, ICSR 2016, pp. 384 390, 2016.

[5] S. Singh, "Clone Detection in UML Class Models using Class Metrics", ACM SIGSOFT Softw. Eng. Notes, Vol. 39, No. 3, pp. 1-4, 2014.

[6] H. Storrle, "Towards Clone Detection in UML Domain Models", Softw. Syst. Model., Vol. 12, No. 2, pp. 307-329, 2013.

[7] J. Kaur and R. Singh, "Implementation of Model Cloning in Software Models using UML diagrams", Int. J. Technol. Comput., Vol. 2, No. 4, pp. 114-119, 2016.

[8] G. D. Kuh, N. Jankowski, S. O. Ikenberry, and J. Kinzie, Knowing what students know and can do: The current state of student learning outcomes assessment in US colleges and universities, No. January. Champaign: National Institute for Learning Outcomes Assessment, 2014.

[9] W. J. Park and D. H. Bae, "A two-stage framework for UML specification matching", Inf. Softw. Technol., Vol. 53, No. 3, pp. 230-244, 2011.

[10] H. O. Salami and M. Ahmed, "A framework for reuse of multi-view UML artifacts", Int. J. Soft Comput. Softw. Eng., Vol. 3, No. 3, pp. 156-162, 2013.

[11] W. K. G. Assuncao and S. R. Vergilio, "Class Diagram Retrieval with Particle Swarm Optimization", In: Proc. of the 25th Software Engineering on International Conf. \& Knowledge Engineering, pp. 632-637, 2013.

[12] K. Robles, A. Fraga, J. Morato, and J. Llorens, "Towards an ontology-based retrieval of UML class diagrams", Inf. Softw. Technol., Vol. 54, No. 1, pp. 72-86, 2012.

[13] D. H. Qiu, H. Li, and J. L. Sun, "Measuring Software Similarity based on Structure and Property of Class Diagram", In: Proc. of Sixth International Conf. on Advanced Computational Intelligence, pp. 75-80, 2013.

[14] M. A.-R. Al-Khiaty and M. Ahmed, "UML Class Diagrams: Similarity Aspects and Matching", Lect. Notes Softw. Eng., Vol. 4, No. 1, pp. 41-47, 2016.

[15] M. A. R. Al-Khiaty and M. Ahmed, "Matching UML class diagrams using a Hybridized Greedy-Genetic algorithm”, In: Proc. of Int. Sci. 
Tech. Conf. Comput. Sci. Inf. Technol., Vol. 1, pp. 161-166, 2017.

[16] M. A.-R. M. Al-Khiaty and M. Ahmed, "Similarity Assessment of UML Class Diagrams using a Greedy Algorithm", In: Proc. of International Computer Science and Engineering Conf., pp. 19-23,2014.

[17] M. A.-R. Al-Khiaty and M. Ahmed, "Similarity assessment of UML class diagrams using simulated annealing", In: Proc. of International Conf. on Software Engineering and Service Science, pp. 19-23, 2014.

[18] A. Adamu and W. M. N. W. Zainon, "Multiview Similarity Assessment Technique of UML Diagrams", Procedia Comput. Sci., Vol. 124, pp. 311-318, 2017.

[19] A. Adamu and W. M. N. W. Zainon, "Similarity Assessment of UML Sequence Diagrams Using Dynamic Programming", In: Proc. of International Visual Informatics Conf., vol. 1, pp. 270-278, 2017.

[20] A. Adamu, W. M. N. Wan Zainon, and S. M. Abdulrahman, "Empirical investigation of UML models matching through different weight calibration", In: Proc. of ACM Int. Conf. Ser., Vol. Part F1479, pp. 167-172, 2019.

[21] Z. Yuan, L. Yan, and Z. Ma, "Structural similarity measure between UML class diagrams based on UCG', Requir. Eng., No. 0123456789, pp. 1-17, 2019.

[22] L. Huang, Y. Duan, X. Sun, Z. Lin, and C. Zhu, "Enhancing UML Class Diagram Abstraction with Knowledge Graph", In: Proc. of International Conf. on Intelligent Data Engineering and Automated Learning, pp. 606616, 2016.

[23] J. Masip, C. Martínez, I. Blandón-Gitlin, N. Sánchez, C. Herrero, and I. Ibabe, "Learning to detect deception from evasive answers and inconsistencies across repeated interviews: A study with lay respondents and police officers", Front. Psychol., Vol. 8, No. JAN, pp. 1-17, 2018.

[24] A. Ylonen, H. Gillespie, and A. Green, "Disciplinary differences and other variations in assessment cultures in higher education: exploring variability and inconsistencies in one university in England", Assess. Eval. High. Educ., Vol. 43, No. 6, pp. 1009-1017, 2018.

[25] J. Moreno and A. F. Pineda, "A Framework for Automated Formative Assessment in Mathematics Courses", IEEE Access, Vol. 8, pp. 30152-30159, 2020.

[26] M. Y. Jebli, R., Elbouhdidi, J., \& Chkouri, "Automatic Evaluation of UML Class Diagrams Using the XML Schema Matching and the
Machine Learning Algorithm", in Advanced Intelligent Systems for Sustainable Development (AI2SD'2018), 2019, Vol. 912, pp. 149-156.

[27] G. Gleb and N. Alexey, "Approach to determining the structural similarity of software projects", Open Semant. Technol. Des. Intellect. Syst., 2018.

[28] R. Fauzan, D. Siahaan, and S. Rochimah, "Class Diagram Similarity Measurement : A Different Approach", In: Proc. of 2018 3rd International Conf. on Information Technology, Information System and Electrical Engineering (ICITISEE), pp. 215-219, 2018.

[29] G. Majumder, P. Pakray, A. Gelbukh, and D. Pinto, "Semantic textual similarity methods, tools, and applications: A survey", Comput. y Sist., Vol. 20, No. 4, pp. 647-665, 2016.

[30] C. D. Manning, J. Bauer, J. Finkel, and S. J. Bethard, "The Stanford CoreNLP Natural Language Processing Toolkit", In: Proc. of 52nd Annual Meeting of the Association for Computational Linguistics: System Demonstrations, pp. 55-60, 2014.

[31] L. J. Garcia Castro, R. Berlanga, and A. Garcia, "In the pursuit of a semantic similarity metric based on UMLS annotations for articles in PubMed Central Open Access", J. Biomed. Inform., Vol. 57, pp. 204-218, 2015.

[32] J. Gao, B. Zhang, and X. Chen, "A WordNetbased semantic similarity measurement combining edge-counting and information content theory", Eng. Appl. Artif. Intell., Vol. 39, pp. 80-88, 2015.

[33] Y. Feng, E. Bagheri, F. Ensan, and J. Jovanovic, "The state of the art in semantic relatedness: a framework for comparison", Knowl. Eng. Rev., pp. 1-30, 2017.

[34] R. Fauzan, D. Siahaan, S. Rochimah, and E. Triandini, "Activity diagram similarity measurement: A different approach", in 2018 International Seminar on Research of Information Technology and Intelligent Systems, ISRITI 2018, pp. 601-605, 2018.

[35] V. Carletti, B. Gaüzère, L. Brun, and M. Vento, "Approximate Graph Edit Distance Computation Combining Bipartite Matching and Exact Neighborhood Substructure Distance", in International Workshop on Graph-Based Representations in Pattern Recognition, pp. 188-197, 2015.

[36] K. Riesen and H. Bunke, "Graph Edit Distance Novel Approximation Algorithms", in Handbook of Pattern Recognition and Computer Vision, University of Birmingham, pp. 275-291, 2016. 
[37] K. Gwet, "Kappa Statistic is not satisfactory for assessing the extent of agreement between raters", Stat. Methods Inter-Rater Reliab. Assessmen, No. 1, pp. 1-5, 2002.

[38] J. Benesty, J. Chen, Y. Huang, and I. Cohen, "Pearson Correlation Coefficient", in Noise reduction in speech ..., Vol. 2, Springer Berlin Heidelberg, p. 229, 2009.

[39] R. T. Lange, "Inter-rater Reliability", in Encyclopedia of Clinical Neuropsychology, Kreutzer, J. S. and DeLuca, J. Caplan, and Bruce, Eds. Springer New York, pp. 1348-1348, 2011.

[40] N. H. Ali, Z. Shukur, and S. Idris, "A Design of an Assessment System for UML Class Diagram Noraida", In: Proc. of - the 2007 International Conference on Computational Science and its Applications, ICCSA 2007, 2007, pp. 539-544, doi: 10.1109/ICCSA.2007.31. 